\title{
INTEGRATING SAFETY CULTURE INTO OSH RISK MITIGATION: A PILOT STUDY ON THE ELECTRICAL SAFETY
}

\author{
Dong ZHAO ${ }^{\mathrm{a}}$, Andrew MCCOY ${ }^{\mathrm{a}}$, Brian KLEINER ${ }^{\mathrm{b}}$, Yingbin $\mathrm{FENG}^{\mathrm{c}}$ \\ ${ }^{a}$ Department of Building Construction, Virginia Polytechnic Institute and State University, \\ Blacksburg, 24061 VA, USA \\ ${ }^{b}$ Myers-Lawson School of Construction, Virginia Polytechnic Institute and State University, \\ Blacksburg, 24061 VA, USA \\ ${ }^{c}$ School of Computing, Engineering and Mathematics, University of Western Sydney, NSW 2751, Australia
}

Received 01 Jul 2013; accepted 19 Sep 2013

\begin{abstract}
Efforts have been taken for years to minimize the occupational safety and health (OSH) risk, but the injury records remain a constant reason for worldwide concerns. Many firms often implement technology as an administrative hierarchy of control (HOC). However, technologies may also actively influence safe practices at the managerial level for administrative HOC. This research examines electrical safety hazards in the U.S. construction industry as a basis for, studying the feasibility of using technology to integrate safety culture into the administrative level of OSH risk mitigation. The researchers introduce the concept of "habitus", which suggests one possibility for establishing a safety culture that increases workers' safety performance and integrates into workers' safety practices through cutting-edge information technology. A prototype application for OSH training based on mobile virtual reality (MVR) technology is demonstrated to help establish habitus in workers' daily practices, and ultimately to mitigate OSH risks at the administrative level of construction projects. Results from a preliminary validation test strongly support human behavior influence and safe work knowledge comprehension by the prototyped application. Although this prototype is demonstrated as a pilot study of electrical safety, the application is not limited to this area and is scalable to other OSH risks.
\end{abstract}

Keywords: occupational safety and health, electrical safety, construction, virtual reality, risk management.

\section{Introduction}

One key component of risk management for industry is occupational safety and health (OSH). Relevant efforts have been taken for years to minimize risk, but OSH injury records, such as electrical injuries and fatalities in construction, remain a constant reason for worldwide concerns. According to the U.S. Bureau of Labor Statistics (BLS), the U.S. construction industry bore a disproportionate share in electrocution statistics (Fig. 1). Between 2003 and 2011, the U.S. construction sector hired approximately $5.05 \%$ of the entire workforce, but was involved in $47.85 \%$ of the electrical fatal injuries. In 2011, the electrocution rate in U.S. construction was 12.2 per million full-time construction workers, which was nearly seven times the average for the all-industry.

Prior research has attempted to provide a good understanding on construction occupational safety and health (Čyras, Jaras 1996) and minimize hazards through risk management. Risk management presents a concept of risk recognition and control in advance, which includes four main processes: risk identification, risk assessment, risk mitigation and risk avoidance (Zavadskas

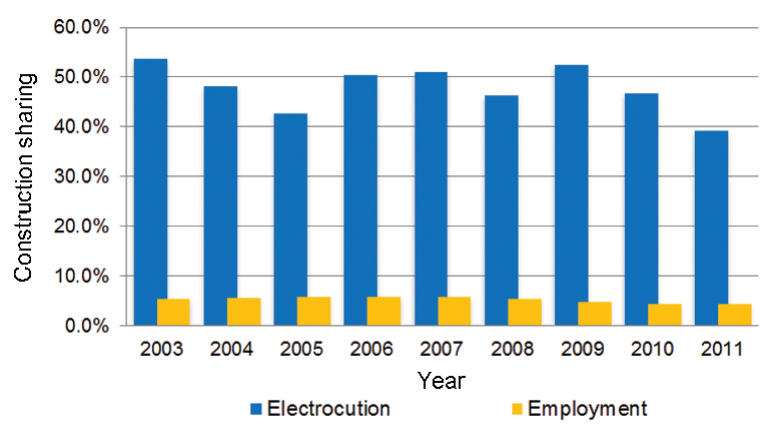

Fig. 1. US Construction's shares in Electrocution and Employment, 2003-2011

Note: data source is U.S. Bureau of Labor Statistics.

et al. 2010). Of these processes, risk occurrence has been considered as a consequence rather than a cause following the action or decision that results in one or more unintended negative outcomes (Strauch 2002). Risk mitigation, the actions and measures applied to reduce the probability of occurrence of an undesirable event and

Corresponding author: Dong Zhao

E-mail:dongz@vt.edu 
its effects, is a preliminary step to risk occurrence and its consequence in terms of risk management. However, most existing research focus on risk assessment and little has focused on mitigation techniques, especially in construction safety (Janicak 2008).

Many firms implement technical systems as risk mitigation techniques for hazards on a construction site. Technical systems offer the ability to actively influence safe practices at the managerial level, part of an administrative hierarchy of control (HOC). This research examines electrical safety hazards in the U.S. construction industry as a basis for studying the feasibility of using technology to integrate safety culture into the administrative level of OSH risk mitigation. Different from purely technical systems as an administrative HOC, this research aims to present an innovative approach to managerial systems for OSH risk mitigation in construction. Researchers in this research introduce the concept of worker "habitus", a type of cultural change that could increase workers' safety performance and integrates into workers' safety practices through cutting-edge information technology. A prototype application for OSH training based on mobile virtual reality (MVR) technology is demonstrated to help establish habitus in workers' daily practices, and ultimately to mitigate OSH risks at the administrative level of construction projects. The researchers conduct a preliminary validation test and results strongly support human behavior influence and safe work knowledge comprehension by the prototyped application.

\section{Electrical OSH risk in construction}

\subsection{Immediate causes of electrocution}

Previous work (Zhao et al. 2014) showed the distribution of electrocution causes in U.S. construction by examining fatality investigations (Fig. 2). Between 1989 and 2011, the main cause of electrical deaths was direct contact with live power lines (accounting for $40 \%$

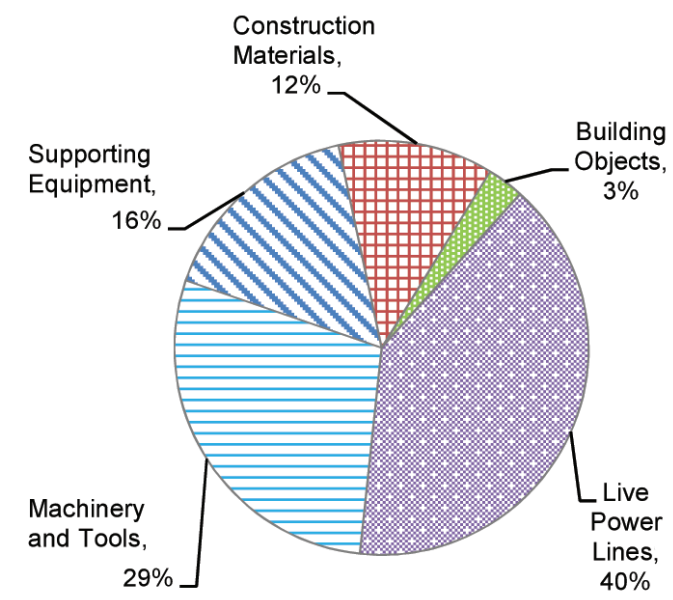

Fig. 2. Immediate causes of electrical deaths in US construction, 1989-2011

Note: the data source is from Zhao et al. (2014). of the incidences). The second-most cause was touching machinery such as cranes, dump trucks or other boomed vehicles. Other causes included contact with energized supporting equipment (e.g. ladders and scaffold) or conductive materials (e.g. pipes, poles).

Victims of electrocution included both electrical workers and non-electrical workers. The electrical occupations include electricians, electrical power installers and repairers, electrical apprentices and helpers, and their supervisors. Only $26 \%$ of victims in electrical deaths were electricians, while the rest were associated with other construction trades (Zhao et al. 2014). Therefore, it seems that not only electrical occupations have been exposed to the electrical hazards, but also non-electrical workers who need increased attention on the electrical hazards and safety.

It is also important to note that a majority of electrocutions noted above had immediate causes of which the industry has knowledge and whose prevention measures have been documented in regulations, codes, and even daily safety procedures. Nevertheless, a large amount of construction workers still get injured or killed annually.

\subsection{Worker behaviors}

Human error has been considered key factor that contributes to up to $80 \%$ of occupational accidents in the aviation, petrochemical, healthcare, construction, mining, and nuclear power industries (Garrett, Teizer 2009). Rasmussen (1997) claimed three types of work performance and associated errors: skill-based, knowledge-based, and rule-based. Reason (2000) combined the knowledge-based and rule-based errors into "human mistakes" and then added further categories for risky behavior in three types: skill-based errors, mistakes and violations. Skill-based error results from limited capacity for information processing, including attention. Mistakes often occur when an individual has either no rules (or "schema") available to apply to a situation or applies the wrong one. Schema are developed through experiential learning, which could occur through repeated exposure to a particular situation, or through learning opportunities provided in training programs. Further, many consider violations different from errors in that they are intentional. As Strauch (2002) summarized, human errors commonly involve failure to identify hazards involved in completing a task or the incorrect use of equipment, while violations are typically related to a failure to follow organizational procedures such as completing a job safety audit or using personal protective equipment. Based on Reason's (2000) three types of risky worker behaviors, human mistakes are considered the major reason for most $\mathrm{OSH}$ incidents.

There may be various reasons for human mistakes, while insufficient training is a significant one. Workers might make mistakes if training fails to pro- 
vide experience in dealing with unfamiliar situations or deliver a broad range of experiences for understanding when rules apply, which lead to flawed interpretations of a situation and poor decision making (Manseau, Shields 2005). Read et al. (2012) utilized a contributing factor framework to verify that human mistakes are associated with a lack of knowledge, task inexperience and deficiencies in training. Jaselskis et al. (1996) argued that unsafe practices often lead to incidents or accidents since work execution is influenced by the lack and less frequency in safety training. In a survey of European countries, Teixeira et al. (2006) concluded that OSH training is needed for construction project management. In other words, an appropriate safety training system could reduce human mistakes as well as various risks in OSH.

\subsection{Safety training practices}

Construction employers typically approach OSH practices for their workforce as part of the overall training orientation within a firm's safety program. Many firms require new workers to participate in the Occupational Safety and Health Administration (OSHA) 30-hour outreach training program for general safety training, which contains an electrical module to train workers of general OSH risk awareness. Site-specific electrical risks are often covered at weekly meetings at each site or during daily hazard meetings.

Within standard training programs, information is presented through slides or the use of video tapes and is reinforced with handouts and study guides (Zhao et al. 2009). Quizzes are also commonly used and included in a training packet to ensure comprehension of the covered material by the trainees.

Outside of the classroom, participatory (i.e. active learning modules) training has been widely accepted as one of the most effective training approaches. As claimed by Goldenhar et al. (2001), the best way for training and learning is to do the real thing and to simulate the real tasks to obtain experience. However, most of current of OSH training practices are not in such participatory format and, as a result, can be less effective. Due to the dangerous work environment of electricity, electrical safety presents an especially difficult problem, as some on-thejob training hazards cannot allow trainees to rehearse electrical tasks in a real situation.

\subsection{Challenges for construction industry}

Construction is a project-based industry. Each project is unique and requires independent stakeholders to collaborate at various stages during the project lifecycle. Each construction project can be considered as a multidisciplinary organization which may not continue to work together once the project is completed (Kamara et al. 2002). Further, electrical contracting is highly connected in nature, meaning that each stakeholder affects, and is highly dependent upon, upstream and downstream work for budget, schedule and safety (Hanna, Haddad 2009). This one-off nature of construction, heavy fragmentation and connective structure makes construction a significantly complex process. Also, construction is labor-intensive with high workforce turnover, which can make risk management more difficult than other industries.

The unique nature of construction industry often constrains the ability for traditional OSH training platforms and formats to be effective. Firstly, while the manufacturing industry has fixed facilities, construction workers are continually moving among different construction sites. Thus it becomes difficult for each construction site to provide a unique training venue for each risk and it remains difficult to schedule multiple assemblies. Secondly, compared to the commonly repeated actions of production lines, every construction project is irreplaceable and constrains work that is often highly specific. Thirdly, training workers is not easy in an industry characterized by small firms with low overhead and high mobility of employees. For example, in the United States, $92 \%$ of the 1.25 million construction companies are less than 20 persons.

\section{OSH risk mitigation: an innovation with safety culture}

Literature suggests that unsafe procedures and violations by workers, such as forgetfulness, negligence and recklessness, are the primary causes leading to OSH injuries (Kletz 2001). There is opportunity to reduce unsafe behaviors through appropriate and effective training, though, even if they cannot be eliminated completely. Goldenhar et al. (2001) highlighted that the most direct way to change statistics in human mistakes was through effective worker training. Neville (1998) suggested that effective training programs could help save large costs by preventing accidents. Effective training not only saves lives but also eliminates the extra indirect costs associated with accident investigations, insurance rates, equipment downtime and repair and productivity losses.

The project-based nature of construction introduces additional barriers to OSH risk management, requiring innovations that provide benefits to the whole industry as opposed to specific risks for individual sites. Toole (1998) defined the innovation process as "application of technology that is new to an organization and that significantly improves the design and construction by decreasing the cost, increasing the performance, and improving the business process". Gann and Salter (2000) found that project-based firms rely upon combining technical expertise from other organizations in order to deliver their own technical capabilities, usually in one-off processes.

In construction trades, OSH innovations are often related to ergonomic engineering to improve health and increase safety. Over time, contractors have looked 
to educational institutions to provide a cadre of skilled construction employees to bring new construction technologies into the workforce. Also, the construction industry is particularly eager in seeking new technologies to control risks in site variability, one-off nature, longevity of warranties and other uncertainty inherent in the built environment (McCoy 2009). To date, most administrative safety controls utilize innovations directly applied to safety improvement in a "technical path": for example, the improvement of personal protective equipment (PPE).

However, "technical-path" administrative control innovations (such as PPE) can only impact the essential OSH stakeholder - personnel - in a passive way (Fig. 3), which may cumulatively lower the effectiveness of innovative technology adoption in OSH risk mitigation. As an example, the rapid adoption of ergonomic, health and safety technologies such as safety harnesses may even discomfort, impede and slow down individual workers, causing these workers to resist the innovation. In contrast, administrative controls that are "managerial-path" innovations relate technology in an indirect way but can also impact workers' OSH adoption in an active manner. In other words, despite the direct adoption of new technologies, the culture innovation is a powerful force that can shape the project's overall effectiveness and construction firm's long-term success (Yitmen 2007). Safety culture unconsciously and consistently influences all aspects of organizational life such as workers' attitude to safety and their decision process during important $\mathrm{OSH}$ activities in construction.

Literature suggests that it is a challenge to integrate new technologies into culture, a set of beliefs, attitudes and values. However it becomes possible when

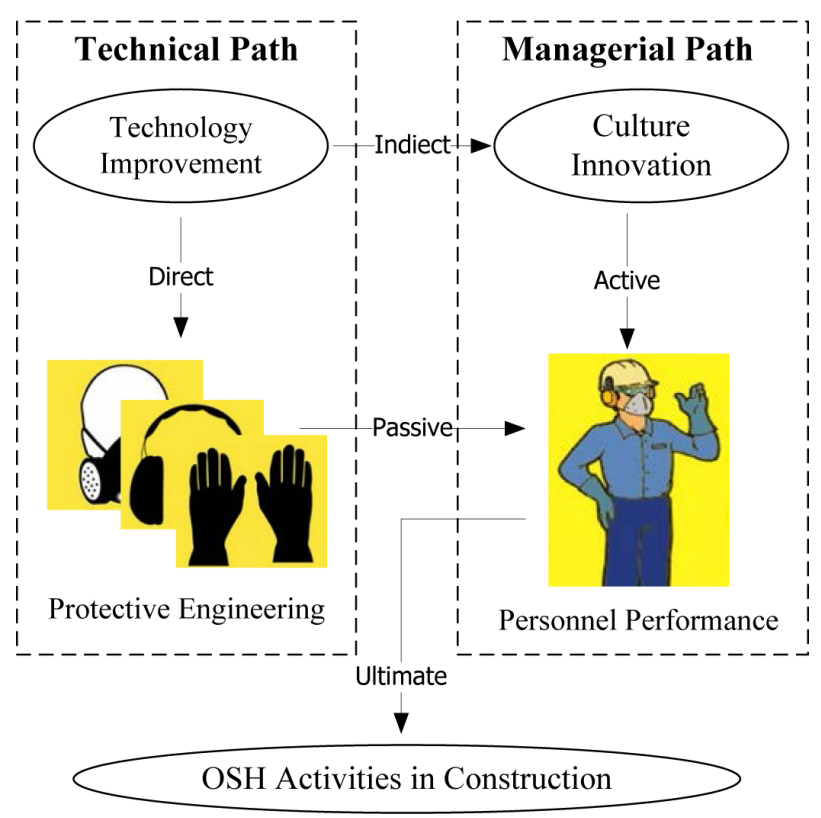

Fig. 3. Managerial path towards OSH risk mitigation culture is associated with the concept of "habitus". In this perspective, culture is not considered a set of beliefs and values but the "whole way of life" which includes practices and routines (Manseau, Shields 2005). Bourdieu (2003) referred to this set of predispositions which guide improvisations in daily routines as the habitus or practical knowledge as repeated routines. One strength in understanding culture as habitus is that routines can be observed and documented, whereas values and beliefs must be inferred, making them less amenable to research. As a result, rather than formulating risk control as a break in habitus, it may prove more useful to conceive of OSH risk mitigation as a process. This process will allow people to show their own propensity toward adoption (decision-adoption process) in an appropriate way, especially when problems are encountered.

Therefore, the habitus, a set of practical routines and dispositions towards certain ways of solving problems, is suggested as an innovative approach to the safetyculture-integrated OSH risk management. Combining risk mitigation as a continuous process of controls, rather than a group of static checkpoints of control, with a habitus-based process of safety training could not only mitigate OSH risk but also complement sustainable productivity and growth for the firm.

\section{A prototype application on electrical safety training}

The following section demonstrates an application of the "habitus" concept for electrical safety training through a virtual reality prototype. The researchers developed the prototype application using mobile virtual reality (MVR) technology to further increase probability of adoption for the changing nature of the industry.

\subsection{Mobile virtual reality technology}

MVR is an adoption of virtual reality (VR) simulation on mobile/portable devices which are connected to cloud technology for end users. It allows safe simulation of real-life events in a digital environment that might otherwise be too dangerous or expensive to create (Haller et al. 1999). VR is described as a 3-dimensional world seen from a first-person view that is under real-time control of the user (Bowman et al. 2005). It also has the ability to create a problem-based learning exercise in an environment that replicates the trainee's actual working environment (McAlpine, Stothard 2003). Training programs via VR offers an interactive, active, and cognitive learning experience for the user (Munro et al. 2002; Stanney, Zyda 2002). As a result, they are often used in place of on-the-job training or full size simulation. Applied to the construction industry, MVR overcomes time and location barriers for workers and provides them more flexibility to access.

MVR also benefits trainees with a participatory training environment. Such participatory training brings 
a real life aspect into the training in an "it can happen to you" scenario and allows the trainees to relate conditions and regulations with real life situations and a lifeor-death importance (Zhao et al. 2009). The best scenario is when people do not have to consciously think about following safety procedures because it is second nature to them (Trybus 2008). Moreover, MVR provide trainees with the ability to experiment without concern for "realworld repercussions" and the ability to "learn by doing." With a MVR program, the user controls the objects and couples this with information and later task-based testing, thus, an interactive and active-learning experience is created.

Most importantly, MVR simulation may contribute to building safety culture in terms of safe practical routines. Through this technology, training programs might allow construction workers to be familiar with common hazards, including dangerous electrical hazards, and to mock up relevant prevention practices without real injury repercussions. It may not only improve trainees' awareness of potential risks in a reality-based working environment, but also unconsciously influence routine behaviors as second nature, which will largely lead to the safety culture.

\subsection{Prototype demonstration}

The researchers developed a prototype of the MVRintegrated OSH training application with an attempt to transfer trainees' safe practices in a virtual world into their routines in real situations. Trainees are expected to be prepared for their future electrical tasks by rehearsing in a virtual environment. The goal of repeated rehearsal is not only to enhance trainees' professional skills but also, more importantly, to help build up their habitus for safe practices.

The training content comprised a variety of construction scenarios in which electrical accidents often occur. The designated scenario was based on the study results from Zhao et al. (2014) which using statistical methods generalized the typical features of electrocution circumstance in the U.S. construction industry. The prototype incorporated these features into one scenario which was a road construction site with overhead power lines surrounded (Fig. 4). The scenario development included two major aspects: environment modeling and storybook coding. The environment modeling simulated construction-related objects and characters while the storybook coding linked these objects and characters with hidden electrical hazards. The modeling and coding was completed using Autodesk 3DS max and Torque 3D package.

Within the prototype, an example of safe practice routine was to conduct a site survey for potential hazards before starting works, as this risk control measure is listed among top five suggestions for preventing electrocution by the National Institute of Occupational Safety and Health (NIOSH). Accident investigations show that this procedure is critical for workers to get hazard awareness

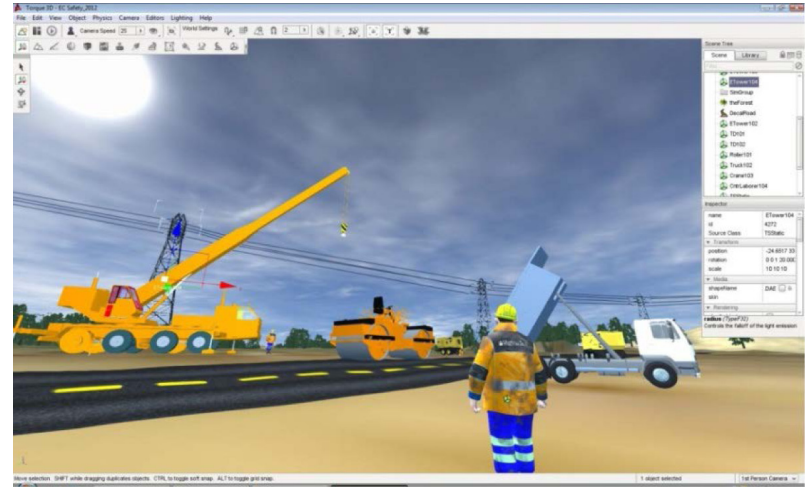

Fig. 4. Prototype application development

but it was often overlooked in real practice. The prototype simulated several site survey actions, some of which were often skipped by workers in real task. For example, workers need to check if the overhead power lines are energized, or to check the safe clearance between machine and electricity source. The application simulated these routine actions in the prototyped scenario, in which users must complete the required site survey procedures by detecting and eliminating the hazards. Following instructions, the user must also repeat the hazard surveying task within specific intervals. The repetition was designed based on memory mechanism and help users to foster best practices of construction safety in their mind. If the user fails, an animation of failure would show up and illustrate the outcome of possible injuries, for example, giving them the feeling of electric shock.

MVR technology overcomes the training limitations on time and location and facilitates the mandatory and effective rehearsals in the virtual world. As a result, it will help establish the concept of safety risk mitigation as habitus in workers' minds and place habitus into the context of real world practices. In this way, knowledge and awareness can be transferred into workers' OSH practical routines - the safety culture.

Although the application aims to be accessible online, server and network settings have not yet been completed for the prototype. While the current prototype has restricted access for the development process, the final application will be available for internet download and wireless utilization. Still, portable features are necessary to fit the fragmented nature of construction projects and the high mobility of construction workers. Therefore, the current prototype version is available on the iPad (Fig. 5) but will be extended to support both iOS and Android platforms.

\section{Preliminary validation}

A preliminary validation test was conducted in a small group of 13 volunteer users. These participants are undergraduate students from the college of engineering at Virginia Tech. The participants were required to complete 


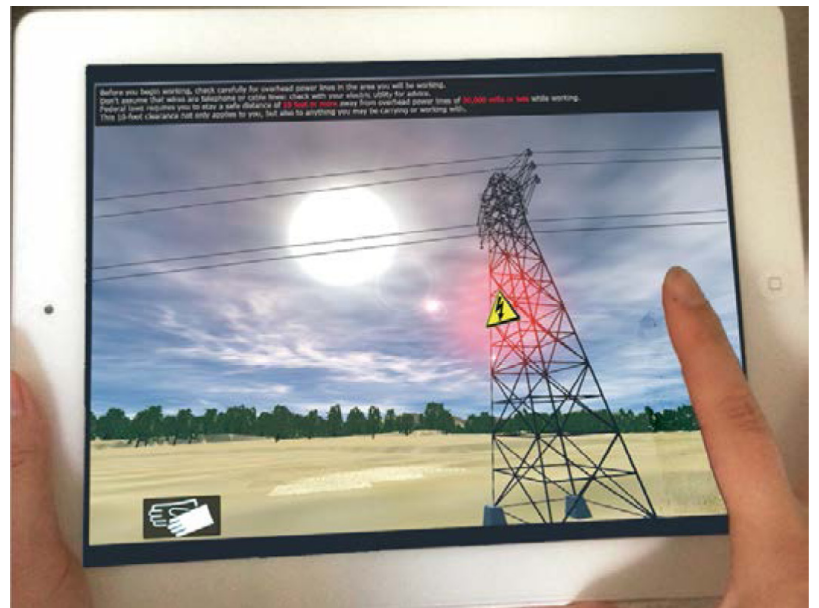

Fig. 5. Prototype interface on iPad

the electrical safety training program following instructions. A round of training lasted for five days, within which respondents would have to consistently repeat OSH training on their mobile devices. The repeat intervals were designed based on a human's curve of forgetfulness and memory mechanisms (Ebbinghaus 1913), which were coded in the application.

After the participant completed the testing routine, a brief survey instrument of ten questions was distributed to them. The objective of the survey was to collect initial prototype feedback and evaluate the application. The ten survey questions all applied a five-point Likert scale in which the five levels were:

1. Strongly disagree;

2. Disagree;
3. Nature;

4. Agree;

5. Strongly agree.

The survey instrument was designed to collect information that would validate four aspects of the application, including application usage, knowledge comprehension, behavior influence and confidence building. Survey results, as summarized in the Table, indicate an overall positive rating with an average score of 4.26 (in a scale of 5). Specifically, it shows a strong agreement on behavior influence for participants, with two scores tying for highest (4.69 for question \#6 and \#8). While a small sample, the analysis suggests that this study's assumption is validated: personnel safety performance can be influenced by training systems that attempt to build a habitus of safety culture. Results also indicate that knowledge comprehension was influenced by the application (question \#3, \#4 and \#5). Participants' confidence when faced with the same safety situation, as presented in the simulation, is fairly supported by the results of question $\# 9$ and \#10. Meanwhile, analysis reveals the imperfection of the application's navigation system as question $\# 1$ received the lowest score of 3.85 , showing an area for improvement in the next prototype version.

While Table 1 represents only a preliminary validation for the prototype application, descriptive statistics show an overall favorability for the MVR technology, its application and adoption in the construction industry.

\section{Conclusions}

One key component of risk management for industry is occupational safety and health (OSH). Relevant efforts have been taken for years to minimize this risk, but OSH

Table 1. Survey results for preliminary validation

\begin{tabular}{lllll}
\hline \multicolumn{1}{c}{ Questions } & \multicolumn{3}{c}{ Responses } \\
\cline { 3 - 5 } & & Highest & Lowest & Mean \\
\hline Application usage & & & \\
\hline $1 \quad$ Do you think the application is comfortable to navigate? & 5 & 3 & 4.85 \\
\hline $2 \quad$ Do you think the application is convenient to access? & & & \\
\hline Knowledge comprehension & 5 & 3 & 4.08 \\
\hline $3 \quad$ Do you think the content clearly present safety knowledge? & 5 & 2 & 4.23 \\
\hline $4 \quad$ Do you think it is better to understand and comprehend? & 5 & 3 & 4.00 \\
\hline $5 \quad$ Do you think you get relevant safe procedure experience? & & & \\
\hline Behavior influence & 5 & 4 & 4.69 \\
\hline $6 \quad$ Do you think VR is appealing to motivate you to continue? & 5 & 3 & 4.38 \\
\hline $7 \quad$ Do you think mandatory repeating help you remember? & 5 & 4 & 4.69 \\
\hline $8 \quad$ Do you think you will apply same practice to real world? & & & \\
\hline Confidence building & $\begin{array}{l}\text { Do you think you will be confident when facing same safety } \\
\text { situation in real world? }\end{array}$ & 5 & 3 & 3.92 \\
\hline & $\begin{array}{l}\text { Do you think you will feel safe when facing same safety } \\
\text { situation in real world? }\end{array}$ & 5 & 4 & 4.38 \\
\hline & Total & 5 & 2 & 4.26 \\
\hline
\end{tabular}


injury records, such as electrocution in construction, remain a reason for worldwide consistent concerns. In most of the efforts for OSH risk mitigation, technologies were often directly used to mitigate hazards and improve safety performance through technical controls that passively influence workers' behavior. However, technologies may also actively influence workers' safe practices at a managerial level. This research examined electrical safety risks in U.S. construction as its basis of study; and analyzed the possibility of integrating safety habitus into the OSH risk mitigation through the use of a mobile virtual reality application.

The researchers' analysis found that a large portion of OSH fatalities and injuries are related to workers' unsafe acts and lack of awareness of OSH hazards. Effective training turns out to be one effective and direct approach to solving this problem but in real life situations, OSH training programs are not effective as they could be. This lack of effectiveness can be attributed to OSH training content as well as the nature of construction industry. Accordingly, the researchers presented an innovative approach to $\mathrm{OSH}$ risk mitigation that is integrated with safety culture. A "safety culture" might directly benefit workers' safety performance and also get forged into workers' safety practices through cutting-edge information technology with the concept of habitus. A prototype application for $\mathrm{OSH}$ training which used mobile virtual reality (MVR) technology was demonstrated to help establish the safety culture in workers' daily practices, and ultimately to mitigate the OSH risks in construction projects. At last, a preliminary validation test was conducted to examine the prototype's performance. The test results strongly supported human behavior influence and safe work knowledge comprehension by the application.

It is important to note that although this OSH risk mitigation application is demonstrated in a pilot study on electrical safety, it is not limited to this industry sector necessarily and could be adapted to other OSH risks. Meanwhile, although this prototype discussion represents only a preliminary validation for the application with utility of MVR, many limitations exist in the evaluation, such as a small sampling pool and a lack of control group for comparing the training effectiveness. Future work will further validate the application's performance in the field of the construction industry with real firms.

\section{References}

Bourdieu, P. 2003. Outline of a theory of practice. New York, NY: Cambridge University Press. 63 p.

Bowman, D. A.; Kruijff, E.; Laviola, J. J. 2005. 3D user interfaces: theory and practice. Boston, MA: Pearson Education, 6-7.

Čyras, P.; Jaras, A. 1996. Occupational safety on construction sites of Lithuania, Statyba 2(8): 110-116. http://dx.doi.org/10.1080/13921525.1996.10590179
Ebbinghaus, H. 1913. Memory: a contribution of experimental psychology. New York City: Columbia Univerity. 123 p.

Gann, D. M.; Salter, A. J. 2000. Innovation in project-based, service-enhanced firms: the construction of complex products and systems, Research Policy 29(7-8): 955-972. http://dx.doi.org/10.1016/S0048-7333(00)00114-1

Garrett, J. W.; Teizer, J. 2009. Human factors analysis classification system relating to human error awareness taxonomy in construction safety, Journal of Construction Engineering and Management 135(8): 754-763.

http://dx.doi.org/10.1061/(ASCE)CO.1943-7862.0000034

Goldenhar, L. M.; Moran, S. K.; Colligan, M. 2001. Health and safety training in a sample of open-shop construction companies, Journal of Safety Research 32(2): 237-252. http://dx.doi.org/10.1016/s0022-4375(01)00045-7

Haller, M.; Kurka, G.; Volkert, J.; Wagner, R. 1999. OmVR a safety training system for a virtual refinery, in ISMCR 99, Topical Workshop on Virtrual Reality and Advanced Human-Robot Systems, 10-11 June 1999, Tokyo, Japan.

Hanna, A. S.; Haddad, G. 2009. Overtime and productivity in electrical construction, Construction Research Congress 2009, 5-7 April 2009, Seattle, Washington, 171-180. http://dx.doi.org/10.1061/41020(339)18

Janicak, C. A. 2008. Occupational fatalities due to electrocutions in the construction industry, Journal of Safety Research 39(6): 617-621. http://dx.doi.org/10.1016/j.jsr.2008.10.007

Jaselskis, E. J.; Anderson, S. D.; Russell, J. S. 1996. Strategies for achieving excellence in construction safety performance, Journal of Construction Engineering and Management 122(1): 61-70.

http://dx.doi.org/10.1061/(ASCE)0733-9364(1996)122:1(61)

Kamara, J. M.; Augenbroe, G.; Anumba, C. J.; Carrillo, P. M. 2002. Knowledge management in the architecture, engineering and construction industry, Construction Innovation 2(1): 53-67. http://dx.doi.org/10.1191/1471417502ci026oa

Kletz, T. A. 2001. Learning from accidents. Oxford, Boston: Gulf Professional Pub. 345 p.

Manseau, A.; Shields, R. 2005. Building tomorrow: innovation in construction and engineering. Burlington, VT: Ashgate. $184 \mathrm{p}$.

McAlpine, I.; Stothard, P. 2003. Using multimedia technologies to support PBL for a course in 3D modeling for mining engineers, in D. Lassner; C. McNaught (Eds.). World Conference on Educational Multimedia, Hypermedia and Telecommunications, 2003, Honolulu, Hawaii, USA, 2449-2455.

McCoy, A. P. 2009. Towards establishing an innovativeness index for construction sites, in Construction Research Congress 2009, 5-7 April 2009, Seattle, Washington, 91-100. http://dx.doi.org/10.1061/41020(339)10

Munro, A.; Breaux, R.; Patrey, J.; Sheldon, B. 2002. Cognitive aspects of virtual evironments design, in K. Stanney (Ed.). Handbook of virtual environments: design, implementation, and applications. Mahwah, NJ: Lawrence Erlbaum Associates, 415-434.

Neville, H. 1998. Workplace accidents: they cost more than you might think, Industrial Management 40(1): 7-9.

Rasmussen, J. 1997. Risk management in a dynamic society: a modelling problem, Safety Science 27(2-3): 183-213. http://dx.doi.org/10.1016/S0925-7535(97)00052-0

Read, G. J. M.; Lenné, M. G.; Moss, S. A. 2012. Associations between task, training and social environmental factors and error types involved in rail incidents and accidents, Accident Analysis \& Prevention 48(0): 416-422. http://dx.doi.org/10.1016/j.aap.2012.02.014

Reason, J. 2000. Human error: models and management, British Medical Journal 320: 768-770. http://dx.doi.org/10.1136/bmj.320.7237.768 
Stanney, K.; Zyda, M. 2002. Virtual environments in the $21^{s t}$ century. Mahwah, New Jersey: Lawrence Erlbaum Associates.

Strauch, B. 2002. Investigating human error: incidents, accidents and complex systems. Burlington, VT, USA: Ashgate. $302 \mathrm{p}$.

Teixeira, J. M. C.; Minasowicz, A.; Zavadskas, E. K.; Ustinovichius, L.; Migilinskas, D.; Armiñana, E. P.; Norwak, P. O.; Grabiec, M. 2006. Training needs in construction project management: a survey of 4 countries of the EU, Journal of Civil Engineering and Management 12(3): 237-245.

Toole, T. 1998. Uncertainty and home builders' adoption of technological innovations, Journal of Construction Engineering and Management 124(4): 323-332.

http://dx.doi.org/10.1061/(ASCE)0733-9364(1998)124:4(323)

Trybus, J. 2008. Making safety second nature: using simulation tools to practice safety, Professional Safety December 54-55.
Yitmen, İ. 2007. The challenge of change for innovation in construction: a North Cyprus perspective, Building and Environment 42(3): 1319-1328. http://dx.doi.org/10.1016/j.buildenv.2005.10.032

Zavadskas, E. K.; Turskis, Z.; Tamošaitiene, J. 2010. Risk assessment of construction projects, Journal of Civil Engineering and Management 16(1): 33-46. http://dx.doi.org/10.3846/jcem.2010.03

Zhao, D.; Lucas, J.; Thabet, W. 2009. Using virtual enironments to support electrical safety awareness in construction, in Proceedings of the 2009 Winter Simulation Conference, IEEE, 13-16 December 2009, Austin, TX, 2679-2690. http://dx.doi.org/10.1109/WSC.2009.5429258

Zhao, D.; Thabet, W.; McCoy, A.; Kleiner, B. 2014. Electrical deaths in the U.S. construction: an analysis of fatality investigations, International Journal of Injury Control and Safety Promotion 21(3): 278-288. http://dx.doi.org/10.1080/17457300.2013.824002

Dong ZHAO. Research Assistant of the Department of Building Construction at Virginia Tech (USA). His research interests include construction safety, construction process simulation and visualization, and green building technologies.

Andrew MCCOY. Director of the Virginia Center for Housing Research and Associate Professor of Building Construction at Virginia Tech (USA). His research involves the diffusion and commercialization of innovative projects in the construction industry.

Brian KLEINER. Professor and Director of the Myers-Lawson School of Construction at Virginia Tech (USA). His research interests focus on human-systems integration or macro-ergonomics for improved safety, health and performance.

Yingbin FENG. Lecturer of School of Engineering at the University of Western Sydney (Australia). His research interests include construction safety and health management, and organizational culture and behaviors. 